\title{
The biological function of m6A demethylase ALKBH5 and its role in human disease
}

\author{
Jinyan Wang ${ }^{1,2 \dagger}$, Jinqiu Wang ${ }^{3 \dagger}$, Quan $\mathrm{Gu}^{4 \dagger}$, Yajun Ma ${ }^{1}$, Yan Yang ${ }^{1}$, Jing Zhu ${ }^{1}$ and Quan'an Zhang ${ }^{1 *}$ (0)
}

\begin{abstract}
Human AlkB homolog H5 (ALKBH5) is a primary m6A demethylase, which is dysregulated and acts as a biological and pharmacological role in human cancers or non-cancers. ALKBH5 plays a dual role in various cancers through regulating kinds of biological processes, such as proliferation, migration, invasion, metastasis and tumor growth. In addition, it takes a great part in human non-cancer, including reproductive system diseases. The underlying regulatory mechanisms of ALKBH5 that relys on m6A-dependent modification are implicated with long non-coding RNA, cancer stem cell, autophagy and hypoxia. ALKBH5 is also an independent prognostic indicator in various cancers. In this review, we summarized the current evidence on ALKBH5 in diverse human cancers or non-cancers and its potential as a prognostic target.
\end{abstract}

Keywords: ALKBH5, m6A, Cancer, Non-cancer, Mechanisms, Prognosis

\section{Background}

Chemical modifications of nucleobases were crucial for inducing changes in protein translation and regulating certain signaling pathways, which subsequently modulated kinds of biological processes. N6-methyladenosine (m6A) modification was first identified in mRNA-enriched RNA fractions in 1974, without any special attention [1]. m6A refered to methylation of the N6 position of adenosine base, which was one of the most prevalent and abundant internal modification in mammalian mRNAs and eukaryotes [2]. In recent years, with the application of high-throughput sequencing for detecting $\mathrm{m} 6 \mathrm{~A}$, the understanding of the inside regulatory mechanisms came to light. m6A modification had been proved to be enriched in near stop codon and 3'untranslated terminal region (UTR) and translate near $5^{\prime}$ UTR in a cap-independent manner [3]. m6A RNA modification of various RNAs, including messenger RNAs (mRNAs) [4],

\footnotetext{
*Correspondence: quananzhang_doctor@163.com

†Jinyan Wang, Jinqiu Wang and Quan Gu contributed equally to this work.

${ }^{1}$ Department of Oncology, Nanjing Jiangning Hospital, The Affiliated Jiangning Hospital of Nanjing Medical University, Nanjing 210000, China

Full list of author information is available at the end of the article
}

microRNAs (miRNAs) [5] and long non-coding RNAs (lncRNAs) [6], was a dynamic and reversible posttranscriptional modification process maintained by three different types of protein complex, including m6A readers, writers, and erasers [7]. This dynamic and reversible nature of the m6A modification made it pivotal in the rapid cellular communication. Recently, substantial progresses had been made in understanding m6A modifications in RNA metabolism, mRNA stability and splicing, translation efficiency, nuclear export, as well as alternative polyadenylation $[8,9]$.

Human AlkB homolog H5 (ALKBH5), was a famous m6A demethylase (also called m6A eraser), which had spawned significant biological and pharmacological interest among researchers [10]. Recently, the effects of ALKBH5 on many biological processes had been demonstrated, including proliferation [11], invasion [12], metastasis [13], ossification [14] and so on. In addition, ALKBH5 was also involved in kinds of cancers or noncancers, such as glioblastoma [15], pancreatic cancer [16], colon cancer [17], breast cancer [18], gastric cancer [19], lung cancer [20], ovarian cancer [21], diabetes [22] and reproductive system diseases [23] (Figs. 1,2).

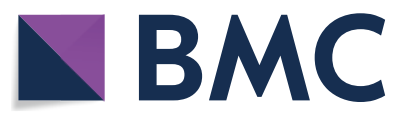

c) The Author(s) 2020. This article is licensed under a Creative Commons Attribution 4.0 International License, which permits use, sharing, adaptation, distribution and reproduction in any medium or format, as long as you give appropriate credit to the original author(s) and the source, provide a link to the Creative Commons licence, and indicate if changes were made. The images or other third party material in this article are included in the article's Creative Commons licence, unless indicated otherwise in a credit line to the material. If material is not included in the article's Creative Commons licence and your intended use is not permitted by statutory regulation or exceeds the permitted use, you will need to obtain permission directly from the copyright holder. To view a copy of this licence, visit http://creativeco mmons.org/licenses/by/4.0/. The Creative Commons Public Domain Dedication waiver (http://creativecommons.org/publicdomain/ zero/1.0/) applies to the data made available in this article, unless otherwise stated in a credit line to the data. 


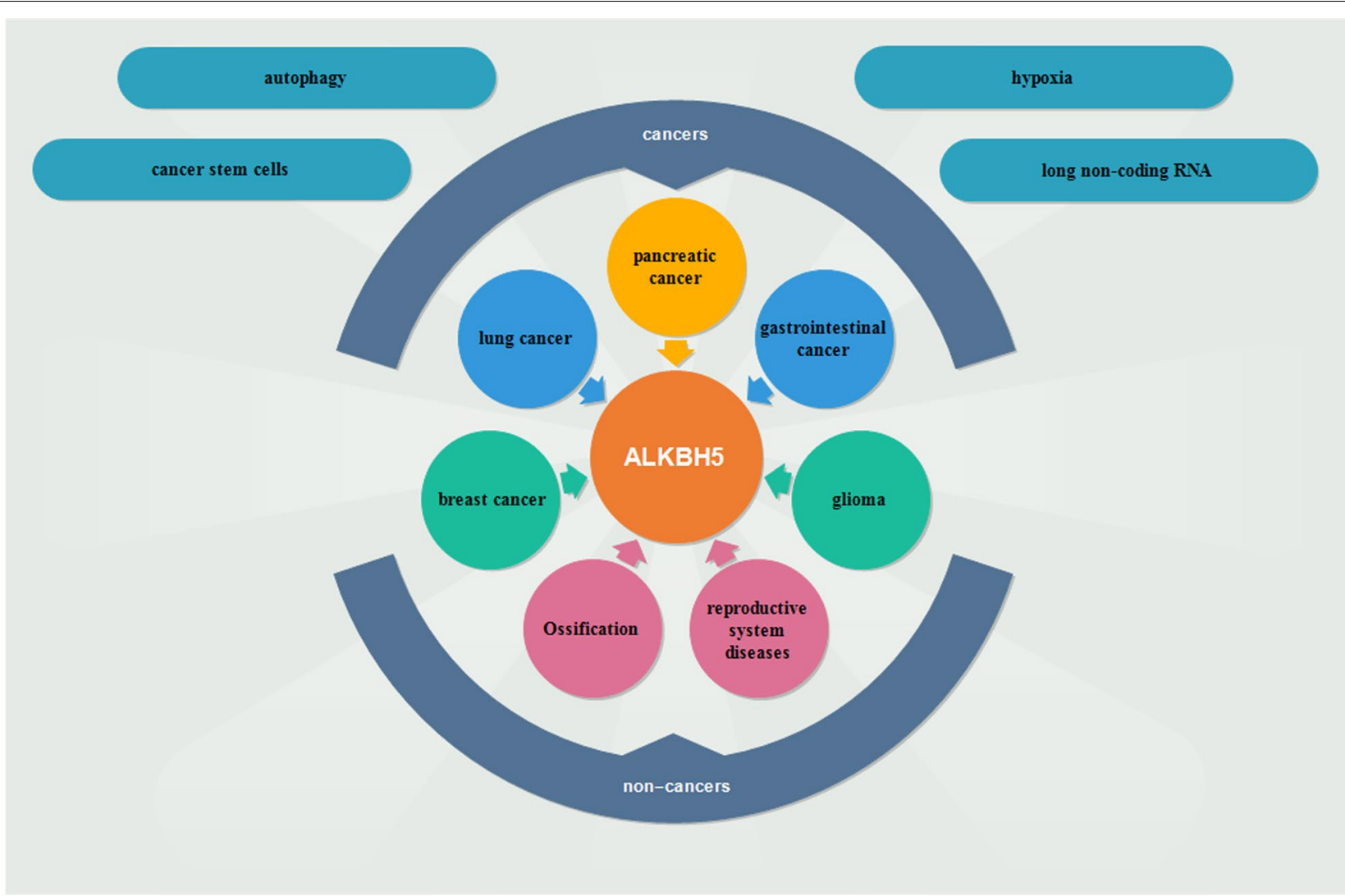

Fig. 1 The mechanisms of ALKBH5 are involved in human cancers and non-cancers. ALKBH5 plays an important role in breast cancer, lung cancer, pancreatic cancer, gastrointestinal cancer and glioma. In addition, ALKBH5 takes a great part in human non-cancers, such as reproductive system diseases and ossification. The underlying mechanisms involve cancer stem cells, autophagy, hypoxia, long non-coding RNA

In the field of cancer research, the underlying mechanisms of ALKBH5 in human cancers were not only unclear but also controversial. Up to date, the expression of ALKBH5 was up-regulated or down-regulated in various cancers, and played an oncogenic or tumor suppressive role in breast cancer, gastric cancer, colon cancer and so on $[17,19,24]$. The potential mechanisms were involved with long non-coding RNAs, miRNAs, mRNA and so on $[19,21,24]$. This kind of communication between ALKBH5 and different RNAs was importantly related with cancer cell proliferation, apoptosis, death, survival, migration, invasion, metastasis and so on $[25,26]$ (Table 1 ).

In the field of human non-cancer research, ALKBH5 was found to be dysregulated in human reproductive system diseases and osteogenic progression [27, 28]. For example, ALKBH5 acted to influence spermatogenesis and trophoblast invasion, thus affecting human reproductive system diseases [23, 29]. In addition, ALKBH5 also played an important role in the ossification of the ligamentum flavum cells and osteogenic progression via some famous signaling pathways, such as AKT and NF- $\mathrm{KB}$ signaling pathway $[14,28]$.

In this study, we summarized the recent advances made in relation to ALKBH5 dysregulation and its biological role coupled with the underlying mechanisms in various human diseases.

\section{The structure of ALKBH5}

ALKBH5 was one of nine members of the AlkB family, a ferrous iron- and 2-oxoglutarate-dependent nucleic acid oxygenase (NAOX) and had been reported to catalyze the demethylation of m6A in RNA [30]. Zhou et al. [30] and Aik et al. [31] identified the crystal structure of ALKBH5 and revealed conserved residues important for recognition and demethylation mechanisms. Chu et al. [32] and Feng et al. [33] respectively identified the crystal structure of zebrafish ALKBH5 (fALKBH5) and human ALKBH5, which promoted the understanding of the substrate recognition specificity of ALKBH5 and offered a foundation for the development of inhibitors selective for NAOX. In addition, Xu et al. [34] affirmed 


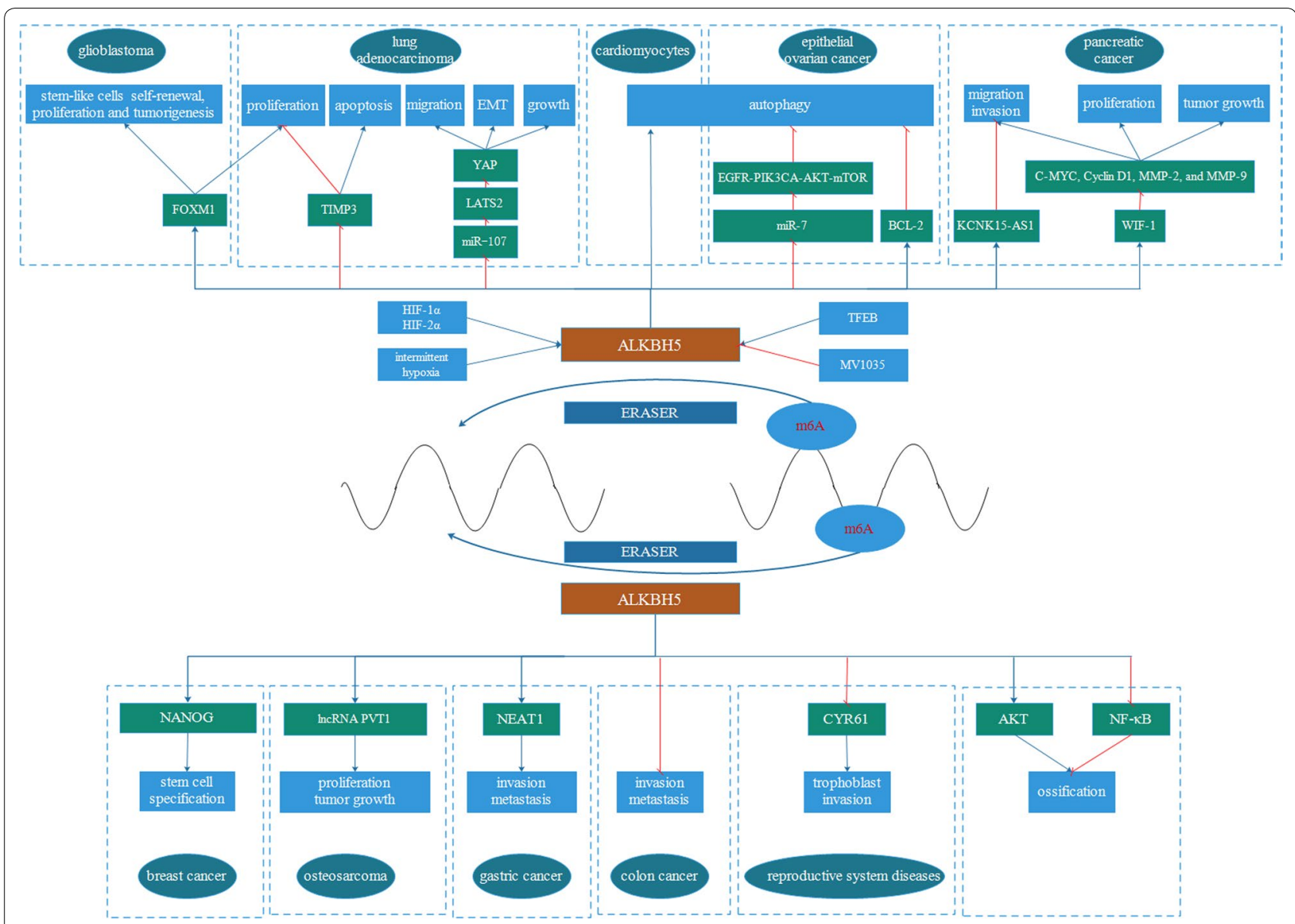

Fig. 2 The mechanisms of ALKBH5 are involved in human cancers and non-cancers. ALKBH5 plays an important role in breast cancer, glioblastoma, overian cancer, lung cancer, pancreatic cancer, gastrointestinal cancer and osteosarcoma, by targeting NANOG, FOXM1, EGFR, BCL-2, NEAT1, FOXM1-AS, KCNK15-AS1, PYT1, TIMP3, YAP, KCNK15-AS1 and WIF-1. The underlying mechanisms involve cancer stem cells, autophagy, hypoxia, long non-coding RNA. In addition, ALKBH5 takes a great part in human non-cancers, such as reproductive system diseases and ossification, by targeting AKT and NF-KB. Blue lines indicate promote and red lines indicate inhibit

Table 1 Expression, clinical significance, and biological function of ALKBH5 in various cancers

\begin{tabular}{|c|c|c|c|c|c|}
\hline Cancer & Expression & Role & Biological Function & Target & References \\
\hline Lung adenocarcinoma & Upregulated & Oncogene & Proliferation and invasion, & FOXM1 & [11] \\
\hline \multirow[t]{2}{*}{ NSCLC } & \multirow[t]{2}{*}{ Upregulated } & \multirow[t]{2}{*}{ Oncogene } & Proliferation, invasion, migration, and EMT & YAP & [13] \\
\hline & & & Proliferation, apoptosis and tumor growth & TIMP3 & [20] \\
\hline \multirow[t]{2}{*}{ Pancreatic cancer } & \multirow[t]{2}{*}{ Downregulated } & \multirow[t]{2}{*}{ Tumor suppressor } & Proliferation, invasion and migration & WIF-1 & [16] \\
\hline & & & Motility & KCNK15-AS1 & [26] \\
\hline Colon cancer & Downregulated & Tumor suppressor & Invasion and metastasis & - & [17] \\
\hline Gastric cancer & - & Oncogene & Invasion and metastasis & NEAT1 & [19] \\
\hline \multirow[t]{2}{*}{ Glioblastoma } & \multirow[t]{2}{*}{ Upregulated } & \multirow[t]{2}{*}{ Oncogene } & Migration and invasion & - & [15] \\
\hline & & & Proliferation, tumorigenesis & FOXM1 & [25] \\
\hline Epithelial ovarian cancer & Upregulated & Oncogene & Proliferation and invasion & $\begin{array}{l}\text { EGFR-PIK3CA- } \\
\text { AKT-mTOR } \\
\text { BCL-2 }\end{array}$ & [21] \\
\hline Clear cell renal cell carcinoma & Downregulated & - & - & - & [45] \\
\hline Osteosarcoma & - & - & Proliferation and tumor growth & PVT1 & [40] \\
\hline Oral squamous cell carcinoma & - & - & Chemoresistance & FOXM1, NANOG & [46] \\
\hline
\end{tabular}


the m6A binding pocket of ALKBH5 and the key residues related with $\mathrm{m} 6 \mathrm{~A}$ recognition through mutagenesis and isothermal titration calorimetry binding experiments. Furthermore, m6A served as a 'conformational marker', which induced different conformational outcomes in RNAs depending on sequence context and critically impacted its interactions with ALKBH5 [35]. In detail, it was found that the DSBH domain of ALKBH5 indispensablely interacted with ATP domain of DDX3, which was a member of the family of DEAD-box RNA helicases and took a great part in key biological processes such as cell cycle, apoptosis, and RNA metabolism [36]. Purslow et al. [37] further investigated the structure and dynamics of ALKBH5 in solution and offered the first atomicresolution model of an AlkB protein in its disordered conformational state. There was also a great connection between single-nucleotide polymorphisms (SNPs) on the two RNA demethylases FTO and ALKBH5 [38]. At the same time, he reported the backbone ${ }^{1} \mathrm{H},{ }^{15} \mathrm{~N},{ }^{13} \mathrm{C}$ chemical shift assignment of a fully active, $26 \mathrm{kDa}$ construct of ALKBH5 [39].

\section{Regulatory mechanisms of ALKBH5 ALKBH5 with long non-coding RNA}

FOXM1-AS, located on chromosome 12(chr12: 29459822968961, GRCh37/hg19), was a nuclear lncRNA that facilitated the communication between ALKBH5 and FOXM1 nascent transcripts. In detail, FOXM1-AS was localized in the same cellular fraction as ALKBH5 and FOXM1 nascent transcripts, and further studies indicated that FOXM1-AS up-regulated FOXM1 expression and played an important role in glioblastoma cells tumorigenesis [25]. Moreover, ALKBH5 demethylated lncRNA KCNK15-AS1, which was downregulated in pancreatic cancer tissues compared with the normal tissues, thus elevating pancreatic cancer motility, including migration and invasion [26]. In addition, ALKBH5 suppressed the degradation of lncRNA plasmacytoma variant translocation 1 (PVT1), which was up-regulated in osteosarcoma tissues and cells, thus promoting the osteosarcoma cell proliferation in vitro and tumor growth in vivo through inhibiting the binding of $\mathrm{m} 6 \mathrm{~A}$ reader YTHDF2 in PVT1 [40]. ALKBH5 decreased the level of lncRNA nuclear paraspeckle assembly transcript 1 (NEAT1), which was overexpressed in gastric cancer cells and tissue, and took a great part in the invasion and metastasis of gastric cancer [19].

\section{ALKBH5 with cancer stem cells}

It was well-known that cancer stem cells were designated as tumor-initiating cells, which were capable of infinite proliferation through self-renewal, giving rise to progeny that were different from them, and forming a secondary (recurrent or metastatic) tumor [41-43]. ALKBH5 promoted breast cancer stem cell (BCSC) specification and enrichment in the tumor microenvironment though catalyzing the demethylation of an adenosine residue in the 3'-UTR of NANOG mRNA [24]. ALKBH5 expression was also elevated in glioblastoma stem-like cells (GSCs), demethylated FOXM1 nascent transcripts and enhanced the expression of FOXM1, thus enhancing stem cells selfrenewal, proliferation and tumorigenesis [25].

\section{ALKBH5 with autophagy}

Another m6A related gene METTL3 relieved autophagic flux in hypoxia/reoxygenation treated cardiomyocytes, however ALKBH5 could reverse this effect [44]. In detail, TFEB activated ALKBH5 transcription through binding to the ALKBH5 promoter. However, the inhibition of METTL3 by TFEB was not dependent on transcriptional repression but rather downregulation of mRNA stability. This negative feedback loop provided insight into the vital link bwtween METTL3-ALKBH5 and autophagy. In addition, the ectopic expression of ALKBH5 inhibited the autophagy of epithelial ovarian cancer cells in vitro and in vivo, partly through activating EGFR-PIK3CAAKT-mTOR signaling pathway, promoting the stability of BCL-2 mRNA, as well as enhancing the interaction between Bcl-2 and Beclin1 [21]. Moreover, ALKBH5 regulated the EGFR expression, relying on HuR and miR-7.

\section{ALKBH5 with hypoxia}

The expression of ALKBH5 was induced by hypoxia in breast cancer cells depending on hypoxia-inducible factor (HIF) $-1 \alpha$ and HIF- $2 \alpha$, and the upregulation of ALKBH5 mediated the enrichment of breast cancer cell in hypoxic tumor microenvironment, through enhancing NANOG expression, therefore advancing tumor formation in vivo [24]. Additionally, TFEB acted to induce the expression of ALKBH5 and controlled the lysosomal-autophagic pathway in hypoxia/re-oxygenation treated cardiomyocytes [44]. Lastly, ALKBH5 was also closely related with the process of intermittent hypoxia in lung adenocarcinoma cells. Mechanistic analysis indicated that m6A demethylase ALKBH5 enhanced the proliferation and invasion of lung adenocarcinoma cells under intermittent hypoxia by down-regulating the level of m6A in FOXM1 mRNA and enhancing the translation efficiency of FOXM1 mRNA, resulting in the overexpression of FOXM1 protein [11].

\section{ALKBH5 dysregulation in human cancers}

In the majority of cancer research, the role of ALKBH5 in human cancers was controversial. It had been found to be upregulated or downregulated, and to play an oncogenic or tumor suppressive role in different kinds of cancers. We will discuss the different roles of ALKBH5 in various 
cancers thereinafter, including breast cancer, lung cancer, pancreatic cancer, glioma and so on.

\section{ALKBH5 in breast cancer}

Recent researches noted that ALKBH5 up-regulated the expression of NANOG by demethylating $\mathrm{m} 6 \mathrm{~A}$ and promoted the gathering of breast cancer cells in the tumor microenvironment $[18,24]$. NANOG, a pluripotency factor, was necessarily required for primary tumor formation and distant metastasis, because of its povital role in the maintenance and specification of cancer stem cells.

\section{ALKBH5 in lung cancer}

ALKBH5 was also elevated in lung adenocarcinoma cells under intermittent hypoxia, and the knockdown of ALKBH5 inhibited the proliferation and invasion of lung adenocarcinoma cells via reducing the $\mathrm{m} 6 \mathrm{~A}$ level of FOXM1 [11]. Additionally, ALKBH5 was obviously upregulated in non-small cell lung cancer (NSCLC) tissues and cell lines, compared with the normal controls, and promoted the malignant biological properties of NSCLC cells via inhibiting the TIMP3 mRNA stability depending on m6A demethylation modification [20]. However, ALKBH5 inhibited tumor growth and metastasis partly through inhibiting the expression and activity of YTHDFs-mediated YAP, and regulating miR-107/LATS2 axis in an HuR-dependent manner [13].

\section{ALKBH5 in gastrointestinal cancer}

ALKBH5 was downregulated in pancreatic cancer cells, and inhibited pancreatic cancer motility [26]. It was found that ALKBH5 sensitized pancreatic ductal adenocarcinoma (PDAC) cells to chemotherapy and ALKBH5 remarkably inhibits pancreatic ductal adenocarcinoma cell proliferation, migration, and invasion, partly by altering the expression of Wnt inhibitory factor 1 (WIF-1), which is mediation of the Wnt pathway [16]. ALKBH5 was downregulated in colon cancer tissues, and the overexpression of ALKBH5 greatly reatrained colon cancer cells invasion in vitro and metastasis in vivo [17]. Besides, ALKBH5 participated in the carcinogenicity of gastric cancer by affecting the methylation of NEAT1 [19].

\section{ALKBH5 in other cancers}

Regarding glioblastoma, ALKBH5 provided a deep insight into critical roles of m6A methylation though enhancing the expression of FOXM1, and promoted tumorigenesis in glioblastoma [25]. And the imidazobenzoxazin-5-thione MV1035 inhibited glioblastoma cell line migration and invasion via inhibiting ALKBH5 [15].

As for other cancers, ALKBH5 was significantly downregulated in clear cell renal cell carcinoma (ccRCC) compared to normal tissue [45]. It was also affirmed that
ALKBH5 took a great part in contributing to osteosarcoma tumorigenesis [40]. ALKBH5, directly regulated by human RNA helicase DDX3, decreased m6A methylation in FOXM1 and NANOG nascent transcript and contributed to chemoresistance of oral squamous cell carcinoma(OSCC) [46]. Besides, ALKBH5 was upregulated in epithelial ovarian cancer tissue compared with the normal ovarian tissues, and enhanced the proliferation and invasion in vitro and in vivo, not only through activating EGFR-PIK3CA-AKT-mTOR signaling pathway, but also through strengthening the stability of BCL-2 mRNA and the interaction between $\mathrm{Bcl}-2$ and Beclin1 [21].

\section{ALKBH5 in cancer prognosis}

Notably, decreased ALKBH5 was closely connected with a shortened overall and cancer-specific survival following nephrectomy in ccRCC [45]. What's more, decreased expression of ALKBH5 was closely associated with metastasis and American Joint Committee on Cancer (AJCC) stage and acted as an independent prognostic indicator in colon cancer patients [17]. Tang, et al. [16] found that low level of ALKBH5 predicted poor clinical outcome in pancreatic ductal adenocarcinoma, phenochromocytoma, paraganglioma, stomach adenocarcinoma and uterine corpus endometrial carcinoma. In detail, the expression of ALKBH5 was also connected with specific clinicopathological features such as TNM staging, tumor size, lymph node metastasis, and distant metastasis. Nontheless, such association could not be observed in breast cancer and head-neck squamous cell carcinoma. Also, Cho, et al. [47] analyzed that ALKBH5 expression was positively associated with overall survival and was a new independent prognostic marker for pancreatic cancer though a retrospective multicohort study.

On the contrary, low level of ALKBH5 was associated with better patients' survival in bladder cancer [16]. Besides, the up-regulated ALKBH5 predicted poor survival in glioblastoma patients [25] and NSLCL patients [20].

The above evidences suggested that the role of ALKBH5 in the prognosis of cancers might be context-dependent.

\section{ALKBH5 dysregulation in human non-cancer}

ALKBH5 was also connected with human non-cancer, such as reproductive system diseases and the process of ossification.

\section{ALKBH5 in reproductive system diseases}

ALKBH5 was the main eraser of the m6A modification in germ cell tumors [48]. ALKBH5 functioned to oxidatively reverse m6A in vitro and vivo, and ALKBH5deficient male mice had increased m6A in mRNA and 
were connected with compromised spermatogenesis through affecting mRNA export and RNA metabolism [29]. Inactivation of ALKBH5 lead to male infertility in mice partly though aberrant splicing and production of shorter transcripts [23]. Moreover, ALKBH5 expression was specifically unregulated in placental villous tissue from recurrent miscarriage patients, and overexpression of ALKBH5 inhibited trophoblast invasion by villous explant culture experiments, through decreasing the half-life of CYR61 mRNA and inhibiting steady-state CYR61 mRNA expression levels [12].

However, it was reported that reduced semen quality exhibited no correlation with genetic variants in the genes coding for the messenger RNA methylation erasers ALKBH5 [27].

\section{ALKBH5 in ossification}

ALKBH5 was increased in ossification of the ligamentum flavum (OLF) tissues, and promoted the ossification of the ligamentum flavum cells through BMP2 demethylation and activating AKT signaling pathway [14]. The METTL3-mediated m6A methylation was found to be dynamically reversed by the demethylase ALKBH5, which acted to reverse the suppression of METTL3 in osteogenic progression, partly though regulating expression of MYD88 and facilitating the activation of NF- $\mathrm{kB}$ which was a well-known repressor of osteogenesis [28].

\section{Conclusion}

The data described in this review suggested the controversial expression patterns and functions of ALKBH5. The mechanisms of ALKBH5 were involved in human cancers and non-cancers. On the one hand, ALKBH5 was up-regulated in kinds of cancer tissues and cell lines, such as breast cancer, lung cancer and epithelial ovarian cancer, and played an oncogenic role in tumor progression. On the other hand, other researchers indicated the opposite conclusions about the expression level and the role of ALKBH5 in pancreatic cancer, colon cancer and clear cell renal cell carcinoma. ALKBH5 regulated cancer cell proliferation, migration, invasion, tumor progression, metastasis, tumorigenesis and chemoresistance through modulating m6A methylation. These effects were orchestrated through multiple mechanisms, such as long non-coding RNAs, cancer stem cells self-renewal and autophagy. ALKBH5 could demethylate lncRNAs, enhance cancer cells self-renewal or regulate autophagy in cancers. Furthermore, ALKBH5 played a great role in human non-cancers, such as reproductive system diseases. Additionally, ALKBH5 had great potential for clinical application by acting as a new prognostic target. However, further researches are still needed to illuminate the exact details of ALKBH5 expression and its mechanisms in human cancers and non-cancers.

\section{Acknowledgements \\ Not applicable.}

\section{Authors' contributions}

(I) Conception and design: JW; (II) Administrative support: YM; (III) Provision of study materials or patients: JW (Jinyan Wang); JW (Jinqiu Wang); QG. (IV) Collection and assembly of data: YY; (V) Data analysis and interpretation: JZ; (VI) Manuscript writing: All authors; (VII) Final approval of manuscript: All authors. All authors read and approved the final manuscript.

\section{Funding}

This work was supported by the Natural Science Foundation of Jiangsu Province (BK20161110 to Quan'an Zhang), the Priority Academic Program Development of Jiangsu Higher Education Institutions (PAPD, JX10231802 to Jinyan Wang), Postgraduate Research and Practice Innovation Program of Jiangsu Province (SJCX17_0387 to Jinyan Wang), the Science Foundation of Jiangsu Health vocational college (JKC201948 to Jinyan Wang), and the Science and Technology Development Fund of Nanjing Medical University (NMUB2019235 to Jinyan Wang), Nanjing health science and Technology Development Fund (YKK18201 to Yan Yang); Science and technology development fund of Nanjing Medical University (2017NJMUZD091 to Yan Yang); and the Research and development fund of Kangda College of Nanjing Medical University (KD2017KYJJYB017 to Yan Yang).

Availability of data and materials

Not applicable.

Ethics approval and consent to participate

Not applicable.

Consent for publication

Not applicable.

Competing interests

The authors declare that they have no competing interests.

\section{Author details}

${ }^{1}$ Department of Oncology, Nanjing Jiangning Hospital, The Affiliated Jiangning Hospital of Nanjing Medical University, Nanjing 210000, China. 2 Department of Oncology, The Affiliated Jiangning Hospital of Jiangsu Health Vocational College, Nanjing 210000, China. ${ }^{3}$ Department of Oncology, Dafeng People's Hospital, Yancheng 224000, China. ${ }^{4}$ Department of Oncology, The Affiliated Cancer Hospital of Nanjing Medical University, Nanjing 210000, China.

Received: 20 May 2020 Accepted: 22 July 2020

Published online: 28 July 2020

\section{References}

1. Desrosiers R, Friderici K, Rottman F. Identification of methylated nucleosides in messenger RNA from Novikoff hepatoma cells. Proc Natl Acad Sci USA. 1974;71:3971-5.

2. Zhang $\mathrm{C}, \mathrm{Fu} J$, Zhou Y. A review in research progress concerning m6A methylation and immunoregulation. Front Immunol. 2019;10:922.

3. Zheng W, Dong $X$, Zhao Y, Wang S, Jiang H, Zhang M, et al. Multiple functions and mechanisms underlying the role of METTL3 in human cancers. Front Oncol. 2019;9:1403.

4. Qian JY, Gao J, Sun X, Cao MD, Shi L, Xia TS, et al. KIAA1429 acts as an oncogenic factor in breast cancer by regulating CDK1 in an N6-methyladenosine-independent manner. Oncogene. 2019;38:6123-41.

5. Chen X, Xu M, Xu X, Zeng K, Liu X, Sun L, et al. METTL14 suppresses CRC progression via regulating N6-methyladenosine-dependent primary miR375 processing. Mol Ther. 2020;28:599-612.

6. Chen X-Y, Zhang J, Zhu J-S. The role of m(6)A RNA methylation in human cancer. Mol Cancer. 2019;18:103. 
7. Zhu S, Wang JZ, Chen D, He YT, Meng N, Chen M, et al. An oncopeptide regulates $m(6)$ A recognition by the $m(6) A$ reader IGF2BP1 and tumorigenesis. Nat Commun. 2020;11:1685.

8. Ma S, Chen C, Ji X, Liu J, Zhou Q, Wang G, et al. The interplay between m6A RNA methylation and noncoding RNA in cancer. J Hematol Oncol. 2019;12:121.

9. Yu J, Chen M, Huang H, Zhu J, Song H, Zhu J, et al. Dynamic m6A modification regulates local translation of mRNA in axons. Nucleic Acids Res. 2018:46:1412-23.

10. Ensfelder TT, Kurz MQ. ALKBH5-induced demethylation of mono- and dimethylated adenosine. Chem Commun. 2018;54:8591-3.

11. Chao Y, Shang J, Ji W. ALKBH5-m(6)A-FOXM1 signaling axis promotes proliferation and invasion of lung adenocarcinoma cells under intermittent hypoxia. BJU Int. 2020;521:499-506.

12. Li XC, Jin F, Wang BY, Yin XJ, Hong W, Tian FJ. The m6A demethylase ALKBH5 controls trophoblast invasion at the maternal-fetal interface by regulating the stability of CYR61 mRNA. Theranostics. 2019;9:3853-65.

13. Jin $\mathrm{D}$, Guo J. m(6)A demethylase ALKBH5 inhibits tumor growth and metastasis by reducing YTHDFs-mediated YAP expression and inhibiting miR-107/LATS2-mediated YAP activity in NSCLC. Mol Cancer. 2020;19:40.

14. Wang HF, Kuang MJ, Han SJ, Wang AB, Qiu J, Wang F, et al. BMP2 modified by the $\mathrm{m}(6) \mathrm{A}$ demethylation enzyme ALKBH5 in the ossification of the ligamentum flavum through the AKT signaling pathway. Calcif Tissue Int. 2020;106(5):486-93.

15. Malacrida A, Rivara M, Di Domizio A, Cislaghi G, Miloso M, Zuliani $V$, et al. 3D proteome-wide scale screening and activity evaluation of a new ALKBH5 inhibitor in U87 glioblastoma cell line. Apoptosis. 2020;28:115300.

16. Tang $B$, Yang $Y$, Kang $M$, Wang $Y$, Wang $Y$, Bi $Y$, et al. m(6)A demethylase ALKBH5 inhibits pancreatic cancer tumorigenesis by decreasing WIF-1 RNA methylation and mediating Wnt signaling. Mol Cancer. 2020;19:3.

17. Yang P, Wang Q, Liu A, Zhu J, Feng J. ALKBH5 holds prognostic values and inhibits the metastasis of colon cancer. Chem Commun.. 2019.

18. Zhang C, Zhi WI, Lu H, Samanta D, Chen I, Gabrielson E, et al. Hypoxiainducible factors regulate pluripotency factor expression by ZNF217- and ALKBH5-mediated modulation of RNA methylation in breast cancer cells. Oncotarget. 2016;7:64527-42.

19. Zhang J, Guo S, Piao HY, Wang Y, Wu Y, Meng XY, et al. ALKBH5 promotes invasion and metastasis of gastric cancer by decreasing methylation of the IncRNA NEAT1. J Physiol Biochem. 2019;75:379-89.

20. Zhou L, Wang Y, Zhu Z, Qian Q, Zhao X, Ma L, et al. N(6)-methyladenosine ALKBH5 promotes non-small cell lung cancer progress by regulating TIMP3 stability. Cancer Cell Int. 2020;731:144348.

21. Zhu H, Gan X, Jiang X, Diao S, Wu H, Hu J. ALKBH5 inhibited autophagy of epithelial ovarian cancer through miR-7 and BCL-2.J Exp Clin Cancer Res. 2019:38:163.

22. Shen F, Huang W, Huang JT, Xiong J, Yang Y, Wu K, et al. Decreased $\mathrm{N}(6)$-methyladenosine in peripheral blood RNA from diabetic patients is associated with FTO expression rather than ALKBH5. FEBS Lett. 2015;100:E148-54.

23. Tang C, Klukovich R, Peng H, Wang Z, Yu T, Zhang Y, et al. ALKBH5dependent m6A demethylation controls splicing and stability of long 3'-UTR mRNAs in male germ cells. Proc Natl Acad Sci USA. 2018;115:E325-33.

24. Zhang C, Samanta D, Lu H, Bullen JW, Zhang H, Chen I, et al. Hypoxia induces the breast cancer stem cell phenotype by HIF-dependent and ALKBH5-mediated $\mathrm{m}^{6} \mathrm{~A}$-demethylation of NANOG mRNA. Autophagy. 2016;113:E2047-56

25. Zhang $\mathrm{S}$, Zhao BS, Zhou A, Lin K, Zheng S, Lu Z, et al. m(6)A demethylase ALKBH5 maintains tumorigenicity of glioblastoma stem-like cells by sustaining FOXM1 expression and cell proliferation program. Cancer Cell. 2017;31(591-606):e6.

26. He Y, Hu H, Wang Y, Yuan H, Lu Z, Wu P, et al. ALKBH5 inhibits pancreatic cancer motility by decreasing long non-coding RNA KCNK15-AS1 methylation. Cell Physiol Biochem. 2018;48:838-46.

27. Landfors M, Nakken S, Fusser M, Dahl JA, Klungland A, Fedorcsak P. Sequencing of FTO and ALKBH5 in men undergoing infertility work-up identifies an infertility-associated variant and two missense mutations. Biomol NMR Assign. 2016;105(1170-9):e5.

28. Wu Y, Yang L, Wang $X$, Du J, Dai J, Chen W, et al. The m6A methyltransferase METTL3 cooperates with demethylase ALKBH5 to regulate osteogenic differentiation through NF-kB signaling. Mol Cancer. 2020;463:203-10.

29. Zheng G, Dahl JA, Niu Y, Fedorcsak P, Huang CM, Li CJ, et al. ALKBH5 is a mammalian RNA demethylase that impacts RNA metabolism and mouse fertility. Mol Cell. 2013;49:18-29.

30. Zhou B, Han Z. Crystallization and preliminary X-ray diffraction of the RNA demethylase ALKBH5. Acta Crystallogr Sect F Struct Biol Cryst Commun. 2013;69:1231-4.

31. Aik W, Scotti JS, Choi H, Gong L, Demetriades M, Schofield CJ, et al. Structure of human RNA N6-methyladenine demethylase ALKBH5 provides insights into its mechanisms of nucleic acid recognition and demethylation. Nucleic Acids Res. 2014;42:4741-54.

32. Chu M, Lin J, Chen W, Zhang L, Zheng G, Fu Y, et al. Crystal structure of the RNA demethylase ALKBH5 from zebrafish. Mol Cell Biochem. 2014;588:892-8.

33. Feng C, Liu Y, Wang G, Deng Z, Zhang Q, Wu W, et al. Crystal structures of the human RNA demethylase Alkbh5 reveal basis for substrate recognition. J Biol Chem. 2014;289:11571-83.

34. Xu C, Liu K, Tempel W, Demetriades M, Aik W, Schofield CJ, et al. Structures of human ALKBH5 demethylase reveal a unique binding mode for specific single-stranded N6-methyladenosine RNA demethylation. J Biol Chem. 2014;289:17299-311.

35. Zou S, Toh JD, Wong KH, Gao YG, Hong W, Woon EC. N(6)-Methyladenosine: a conformational marker that regulates the substrate specificity of human demethylases FTO and ALKBH5. Sci Rep. 2016;6:25677.

36. Shah A, Rashid F, Awan HM, Hu S, Wang X, Chen L. The DEAD-box RNA helicase DDX3 interacts with m(6)A RNA demethylase ALKBH5. Stem Cells Int. 2017;2017:8596135.

37. Purslow JA, Nguyen TT, Egner TK, Dotas RR, Khatiwada B, Venditti V. Active site breathing of human Alkbh5 revealed by solution NMR and accelerated molecular dynamics. Biophys J. 2018;115:1895-905.

38. Piette ER, Moore JH. Identification of epistatic interactions between the human RNA demethylases FTO and ALKBH5 with gene set enrichment analysis informed by differential methylation. Andrology. 2018;12:59.

39. Purslow JA, Venditti V. (1)H, (15)N, (13)C backbone resonance assignment of human Alkbh5. Biomol NMR Assign. 2018;12:297-301.

40. Chen S. ALKBH5-mediated m(6)A demethylation of InCRNA PVT1 plays an oncogenic role in osteosarcoma. Stem Cells Int. 2020;20:34.

41. Nassar D, Blanpain C. Cancer stem cells: basic concepts and therapeutic implications. Annu Rev Pathol. 2016;11:47-76.

42. Nio K, Yamashita T, Kaneko S. The evolving concept of liver cancer stem cells. Mol Cancer. 2017;16:4.

43. O'Conor CJ, Chen T, González I, Cao D, Peng Y. Cancer stem cells in triplenegative breast cancer: a potential target and prognostic marker. Biomark Med. 2018;12:813-20.

44. Song H, Feng X. METTL3 and ALKBH5 oppositely regulate m(6)A modification of TFEB mRNA, which dictates the fate of hypoxia/reoxygenationtreated cardiomyocytes. Autophagy. 2019;15:1419-37.

45. Strick A, von Hagen F, Gundert L, Klümper N, Tolkach Y, Schmidt D, et al. The N(6)-methyladenosine (m(6) A) erasers alkylation repair homologue 5 (ALKBH5) and fat mass and obesity-associated protein (FTO) are prognostic biomarkers in patients with clear cell renal carcinoma. BJU Int. 2020;125:617-24

46. Shriwas O, Priyadarshini M, Samal SK, Rath R, Panda S, Das Majumdar SK, et al. DDX3 modulates cisplatin resistance in OSCC through ALKBH5mediated m(6)A-demethylation of FOXM1 and NANOG. J Clin Lab Anal. 2020;25(3-4):233-46.

47. Cho SH, Ha M, Cho YH, Ryu JH, Yang K, Lee KH, et al. ALKBH5 gene is a novel biomarker that predicts the prognosis of pancreatic cancer: a retrospective multicohort study. Ann Hepatobiliary Pancreat Surg 2018;22:305-9.

48. Nettersheim D, Berger D, Jostes S, Kristiansen G, Lochnit G, Schorle H. N6-Methyladenosine detected in RNA of testicular germ cell tumors is controlled by METTL3, ALKBH5, YTHDC1/F1/F2, and HNRNPC as writers, erasers, and readers. Andrology. 2019;7:498-506.

\section{Publisher's Note}

Springer Nature remains neutral with regard to jurisdictional claims in published maps and institutional affiliations. 